QUARTERLY OF APPLIED MATHEMATICS

VOLUME LXVIII, NUMBER 4

DECEMBER 2010, PAGES 765-781

S 0033-569X(2010)01208-9

Article electronically published on September 17, 2010

\title{
HYPERBOLIC CONSERVATION LAWS WITH LARGE INITIAL DATA. IS THE CAUCHY PROBLEM WELL-POSED?
}

\author{
BY
}

\author{
CHARIS TSIKKOU
}

Department of Mathematics, Brown University, Providence, RI 02912

Abstract. We present an example in which the Glimm estimate for a strictly hyperbolic system of two conservation laws is violated.

1. Introduction. Consider the Cauchy problem for a strictly hyperbolic system of conservation laws in one space dimension:

$$
\left\{\begin{array}{l}
U_{t}+F(U)_{x}=0, \quad-\infty<x<\infty, \quad 0 \leq t<\infty \\
U(x, 0)=U_{0}(x), \quad-\infty<x<\infty
\end{array}\right.
$$

It is known (Glimm [6], Liu [13, Bressan [3], Bianchini and Bressan [2]) that when

$$
T V_{(-\infty, \infty)} U_{0}(\cdot)<\delta
$$

for a small positive constant $\delta$, then there exists an admissible unique, globally defined solution $U(x, t)$ in the class $B V$ of functions of bounded variation. Furthermore,

$$
T V_{(-\infty, \infty)} U(\cdot, t) \leq C T V_{(-\infty, \infty)} U_{0}(\cdot),
$$

where $C$ is a constant depending solely on $F$. The major open problem is whether global existence of a weak solution and the fundamental estimate (1.3) on the total variation hold under the weaker assumption that the total variation, or even the oscillation, of the initial data is large.

Existence for arbitrarily large initial data has been established for scalar conservation laws (Kruzkov [12]). In general, the estimate (1.3), as it stands, is not valid when the total variation of the initial data is large, as Jenssen [10] provided a counterexample showing blow up for certain $3 \times 3$-systems of strictly hyperbolic conservation laws, in which repeated collisions of shocks drive the oscillation or the total variation of solutions to infinity in finite time. It is important to identify classes of systems for which the estimate

Received July 18, 2009.

2000 Mathematics Subject Classification. Primary 35L65.

Key words and phrases. Nonlinear PDE, hyperbolic systems of conservation laws, Glimm-Lax estimates. The author is indebted to her advisor, Professor Constantine M. Dafermos, for his constant help, advice, motivation and patience during her graduate student life at Brown University.

E-mail address: Charis.Tsikkou@mis.mpg.de 


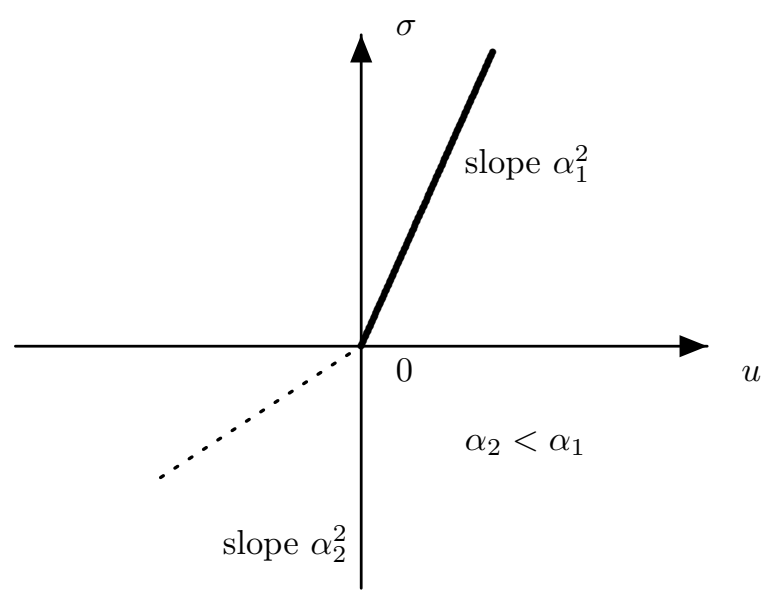

FIG. 1.1. Piecewise linear $\sigma(\cdot)$.

(1.3) is valid. For genuinely nonlinear, strictly hyperbolic systems of two conservation laws, Glimm and Lax [7] showed existence of solutions to the Cauchy problem under initial data with small oscillation but not necessarily small total variation. Assuming that

$$
\sup _{(-\infty, \infty)}|U(\cdot, 0)| \leq \delta
$$

and

$$
T V_{(-\infty, \infty)} U(\cdot, 0)<\alpha \delta^{-\frac{1}{2}}
$$

where $\delta, \alpha$ are small constants, the solution satisfies (1.3) and

$$
T V_{L} U(x, t) \leq \frac{c L}{t},
$$

where $T V_{L}$ stands for the total variation over any interval of length $L$, with $L \geq t$. Similar estimates are derived in Dafermos [4] and Trivisa [16] by the method of generalized characteristics.

However, the case of initial data with large oscillation is still open. In order to gain a better understanding of this problem, we consider the initial value problem

$$
\begin{cases}u_{t}-v_{x}=0, & -\infty<x<\infty, \quad 0 \leq t<\infty ; \\ v_{t}-\sigma(u)_{x}=0, & -\infty<x<\infty, \quad 0 \leq t<\infty ; \\ (u(x, 0), v(x, 0))=\left(u_{0}(x), v_{0}(x)\right), & -\infty<x<\infty,\end{cases}
$$

where $\sigma^{\prime}(u)>0$. We consider the simple case where $\sigma(\cdot)$ is piecewise linear; that is

$$
\sigma(u)= \begin{cases}\alpha_{1}^{2} u, & \text { if } u>0 ; \\ \alpha_{2}^{2} u, & \text { if } u<0,\end{cases}
$$

with $\alpha_{1}>\alpha_{2}>0$, as in Figure 1.1. We show that it is possible to find a sequence $\left\{\left(u_{0 n}, v_{0 n}\right)\right\}$ of initial data with $T V u_{0 n}+T V v_{0 n}$ uniformly bounded, with respect to $n$, for which the estimate (1.3) is violated for any $C$. The reason is that, because of our choice of $\sigma$, one may encounter changes in the characteristic speed of order one even when the change in $u$ is arbitrarily small. The form of the system (1.7) for that particular 
choice of $\sigma$ allows one to treat large data as small. We feel that this example indicates that even when $\sigma(\cdot)$ is smooth, the estimate (1.3) may fail to hold if the oscillation of the initial data is large. The results obtained here shall provide insight on this open problem.

2. Preliminaries. Standard information on the theory of hyperbolic systems of conservation laws is found in the books by Dafermos [4], Holden and Risebro [8], and Smoller [15.

For the system (1.7), the characteristic speeds are $\lambda_{1}=-\sqrt{\sigma^{\prime}(u)}, \lambda_{2}=\sqrt{\sigma^{\prime}(u)}$ and the Riemann invariants are $r(u, v)=v-\int_{0}^{u} \sqrt{\sigma^{\prime}(\theta)} d \theta, s(u, v)=v+\int_{0}^{u} \sqrt{\sigma^{\prime}(\theta)} d \theta$. Throughout the paper, we will be using bold and dotted lines to depict, respectively, jump contact discontinuities with characteristic speeds $\pm \alpha_{1}$ and $\pm \alpha_{2}$. Thin lines will depict compressive shocks with speeds $\pm \lambda$, where $\alpha_{2}<\lambda<\alpha_{1}$, as shown in Figures 2.1, 2.2. and 2.3. The 1-Riemann invariant $r$ remains constant along jump discontinuities with slope $-\left(\alpha_{1}\right)^{-1}$ or $-\left(\alpha_{2}\right)^{-1}$, and the 2-Riemann invariant $s$ remains constant along jump discontinuities with slope $\left(\alpha_{1}\right)^{-1}$ or $\left(\alpha_{2}\right)^{-1}$, as depicted in Figures 2.1 and 2.2. By contrast, $r$ and $s$ vary along 1-shocks with slope $-\lambda^{-1}$, and 2-shocks with slope $\lambda^{-1}$.
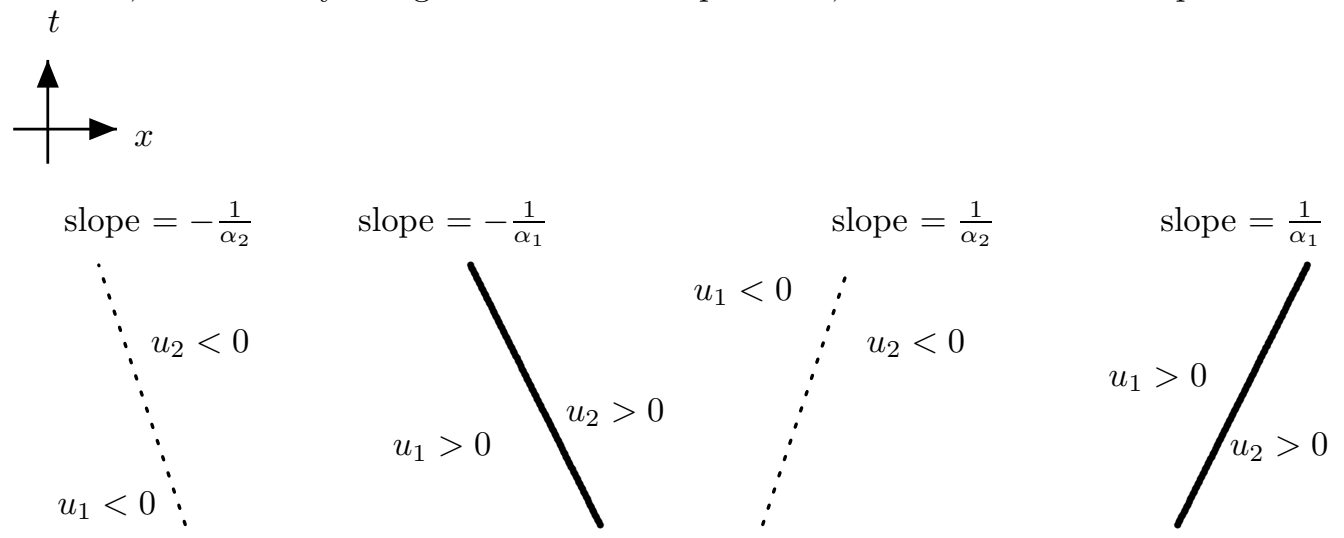

FIG. 2.1. Left-propagating waves.

FIG. 2.2. Right-propagating waves.
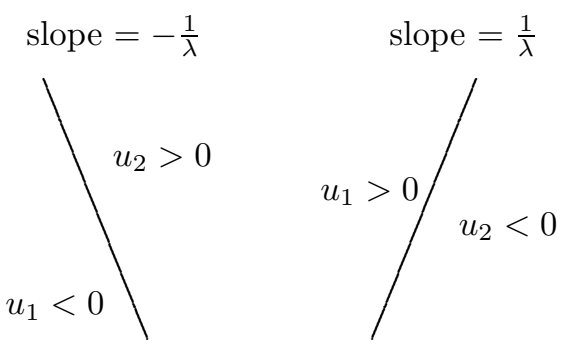

FIG. 2.3. 1- and 2-shocks.

Self-similar solutions to the Riemann problems are generally obtained with the help of shock and rarefaction curves. In our case, since $\sigma$ is piecewise linear, we do not have centered rarefaction waves but only jump discontinuities. Jump discontinuities with 
negative slope, connecting states $\left(u_{1}, v_{1}\right)$ and $\left(u_{2}, v_{2}\right)$, will be called shocks when $u_{1}<u_{2}$ or rarefactions when $u_{1}>u_{2}$. Jump discontinuities with positive slope, connecting states $\left(u_{1}, v_{1}\right)$ and $\left(u_{2}, v_{2}\right)$, will be called shocks when $u_{1}>u_{2}$ or rarefactions when $u_{1}<u_{2}$. The states that can be connected to $\left(u_{-}, v_{-}\right)$by a 1-shock or 2-shock lie on the curve

$$
S_{1}: v-v_{-}=\sqrt{\left(u-u_{-}\right)\left(\sigma(u)-\sigma\left(u_{-}\right)\right)} \equiv s_{1}\left(u ;\left(u_{-}, v_{-}\right)\right), \quad u>u_{-}
$$

or

$$
S_{2}: v-v_{-}=\sqrt{\left(u-u_{-}\right)\left(\sigma(u)-\sigma\left(u_{-}\right)\right)} \equiv s_{2}\left(u ;\left(u_{-}, v_{-}\right)\right), \quad u<u_{-} .
$$

The states that can be connected to the state $\left(u_{-}, v_{-}\right)$by a 1 - or a 2 -rarefaction lie on the curve

$$
R_{1}: v-v_{-}=\int_{u_{-}}^{u} \sqrt{\sigma^{\prime}(y)} d y \equiv \theta_{1}\left(u ;\left(u_{-}, v_{-}\right)\right), \quad u_{-}>u
$$

or

$$
R_{2}: v-v_{-}=-\int_{u_{-}}^{u} \sqrt{\sigma^{\prime}(y)} d y \equiv \theta_{2}\left(u ;\left(u_{-}, v_{-}\right)\right), \quad u>u_{-} .
$$

These curves are depicted in Figure 2.4

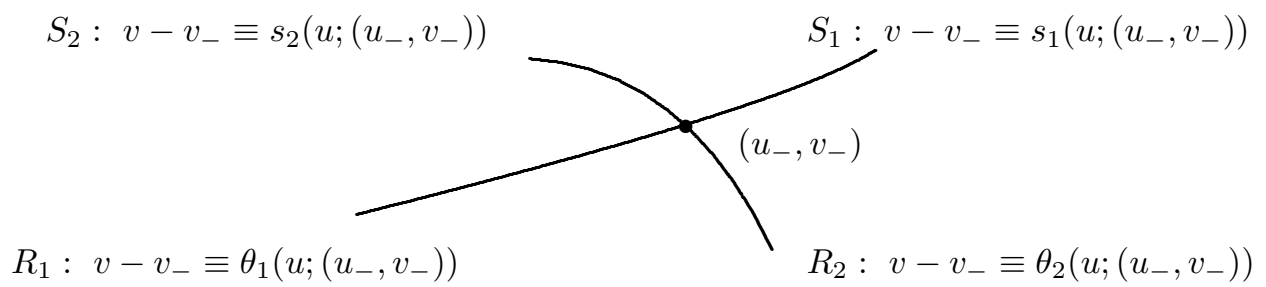

FIG. 2.4. Shock and rarefaction curves.

The solution to the Riemann Problem, with left state $\left(u_{-}, v_{-}\right)$and right state $\left(u_{+}, v_{+}\right)$, has one of the following forms, depending on the sign of the end states, as seen in Figure 2.5.
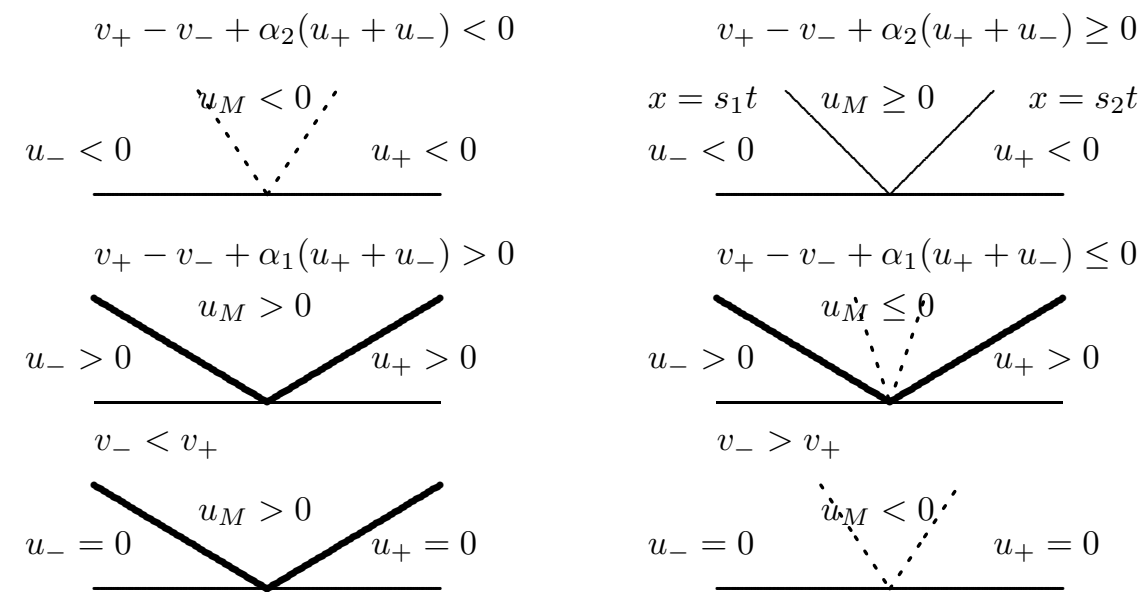

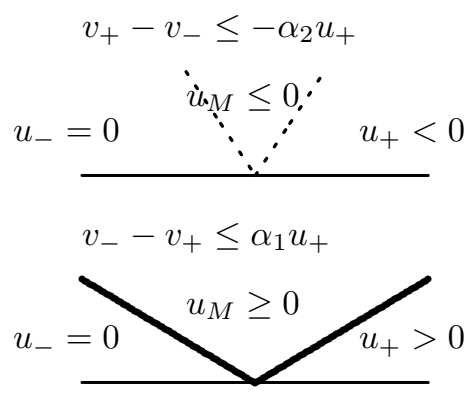

$\alpha_{1} u_{-} \geq v_{-}-v_{+}$

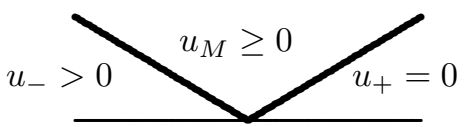

$v_{+}-v_{-} \leq-\alpha_{2} u_{-}$
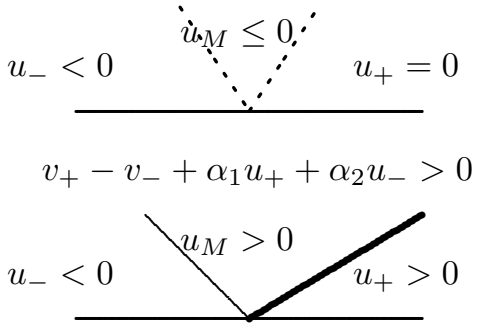

$v_{+}-v_{-}+\alpha_{1} u_{-}+\alpha_{2} u_{+}>0$

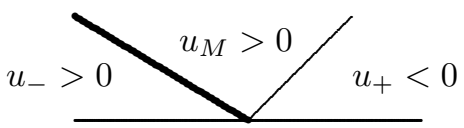

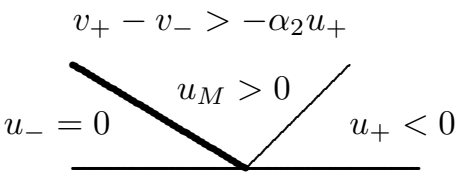

$v_{-}-v_{+}>\alpha_{1} u_{+}$
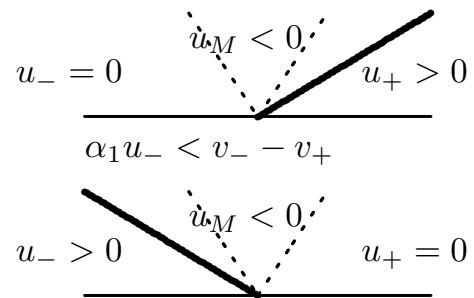

$v_{+}-v_{-}>-\alpha_{2} u_{-}$

$u_{-}<0$
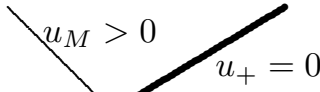

$v_{+}-v_{-}+\alpha_{1} u_{+}+\alpha_{2} u_{-} \leq 0$

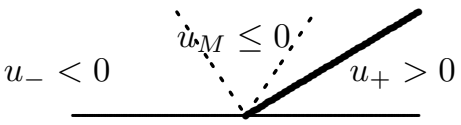

$v_{+}-v_{-}+\alpha_{1} u_{-}+\alpha_{2} u_{+} \leq 0$

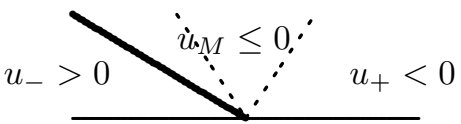

Fig. 2.5. Solutions to the Riemann Problem.
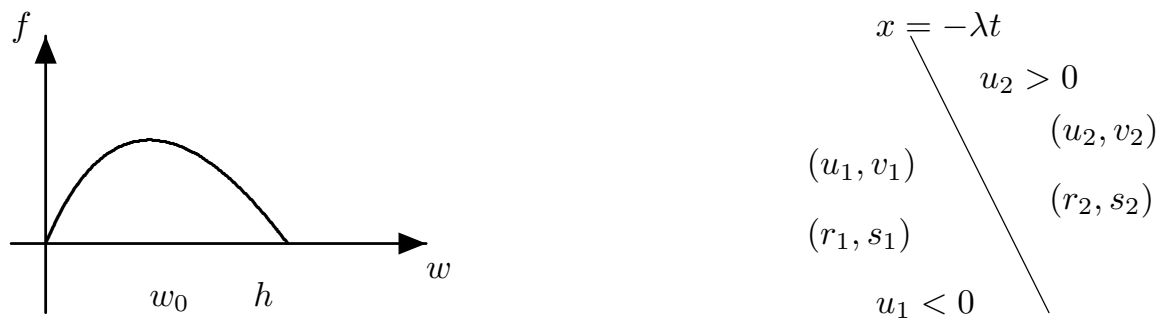

Fig. 2.6. Jump $f=\Delta r\left(\cdot, r_{1}-s_{1}\right)$ of a 1-shock.

Figure 2.6 depicts a 1-shock, which connects the states $\left(u_{1}, v_{1}\right)$ and $\left(u_{2}, v_{2}\right)$ with $u_{1}<0, u_{2}>0$. The speed of the shock is $-\lambda$ where $\alpha_{2}<\lambda<\alpha_{1}$. The corresponding 
Riemann invariants are $\left(r_{1}, s_{1}\right)$ and $\left(r_{2}, s_{2}\right)$. We express the jump in $r, \Delta r=r_{2}-r_{1}$, in terms of the jump in $s, \Delta s=s_{2}-s_{1}$, and $r_{1}-s_{1}$; namely,

$$
\Delta r=f\left(\Delta s, r_{1}-s_{1}\right)
$$

where

$$
f(h, w)= \begin{cases}\frac{2 k w(h-w)}{h+2 k w}, & 0<w<h ; \\ 0, & w \geq h \text { or } w \leq 0,\end{cases}
$$

and

$$
\frac{1}{2 k}=\frac{4 \alpha_{1} \alpha_{2}}{\left(\alpha_{1}-\alpha_{2}\right)^{2}} .
$$

It should be noted that $k \rightarrow 0$ as $\left(\alpha_{1}-\alpha_{2}\right) \rightarrow 0$. The form of the function $f$, depicted in Figure 2.6, will be very useful for our purposes.

If we solve the system (1.7) with $\sigma$ piecewise linear, and $\sigma^{\prime}$ has more than one jump point, then nothing changes, as we can find again a solution with uncontrollable growth. In Tsikkou [18, we recover the Glimm estimate for smooth fluxes by approximating the smooth function $\sigma$ by piecewise linear functions $\sigma_{n}(\cdot)$. This does not contradict the results of the present paper, because as the approximations get tighter, that is, modifying $\sigma_{n}$ in a small neighborhood of the jump points of $\sigma_{n}^{\prime}$ so that $\sigma$ is in $C^{1}$, the jumps in the slopes of $\sigma_{n}(\cdot)$ and thereby the $k$ corresponding to each such jump get smaller.

3. Solutions with uncontrollable growth. It is more convenient to use the vector $(r, s)$ of Riemann invariants in the place of the original state vector $(u, v)$. The goal is to show that there are wave interactions inducing increase in the total variation that violates the estimate

$$
T V r(\cdot, t)+T V s(\cdot, t) \leq C\left\{T V r_{0}(\cdot)+T V s_{0}(\cdot)\right\}
$$

for any $C$. For that purpose, we construct a sequence of initial data $\left(r_{0 n}, s_{0 n}\right)$ with $T V r_{0 n}(\cdot)+T V s_{0 n}(\cdot)=1$, for every $n$, and show that the sequence of solutions $\left(r_{n}, s_{n}\right)$ satisfies $T V r_{n}\left(\cdot, t_{n}\right)+T V s_{n}\left(\cdot, t_{n}\right)>c_{n}$, where $c_{n} \rightarrow \infty$. To define the sequence of initial data, we will use as parameters the positive numbers $\alpha, \beta, b, L, Q$ and the two positive integers $M, N$. We introduce two step functions, $\chi(x ; \alpha, b, L, M)$ and $\psi(x ; \beta, b, Q, N)$, depicted in Figure 3.1 defined by

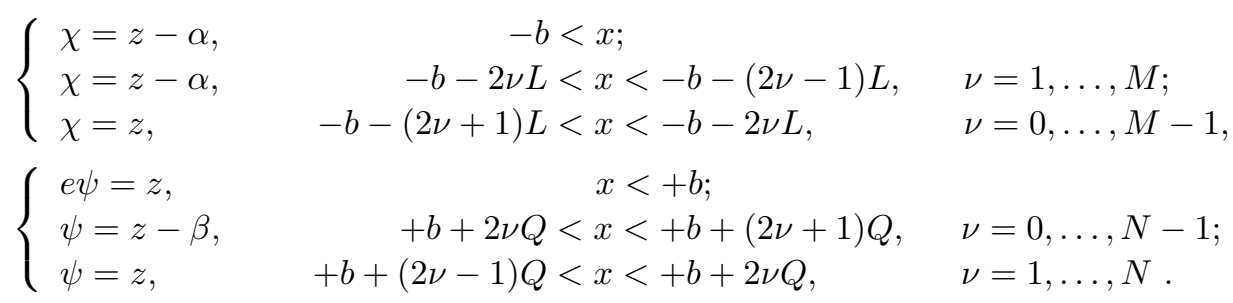

The function $\chi$ is a step function with $2 M$ jumps on the negative semi-axis and constant on the positive semi-axis. The function $\psi$ is a step function with $2 N$ jumps on the positive semi-axis and constant on the negative semi-axis. 


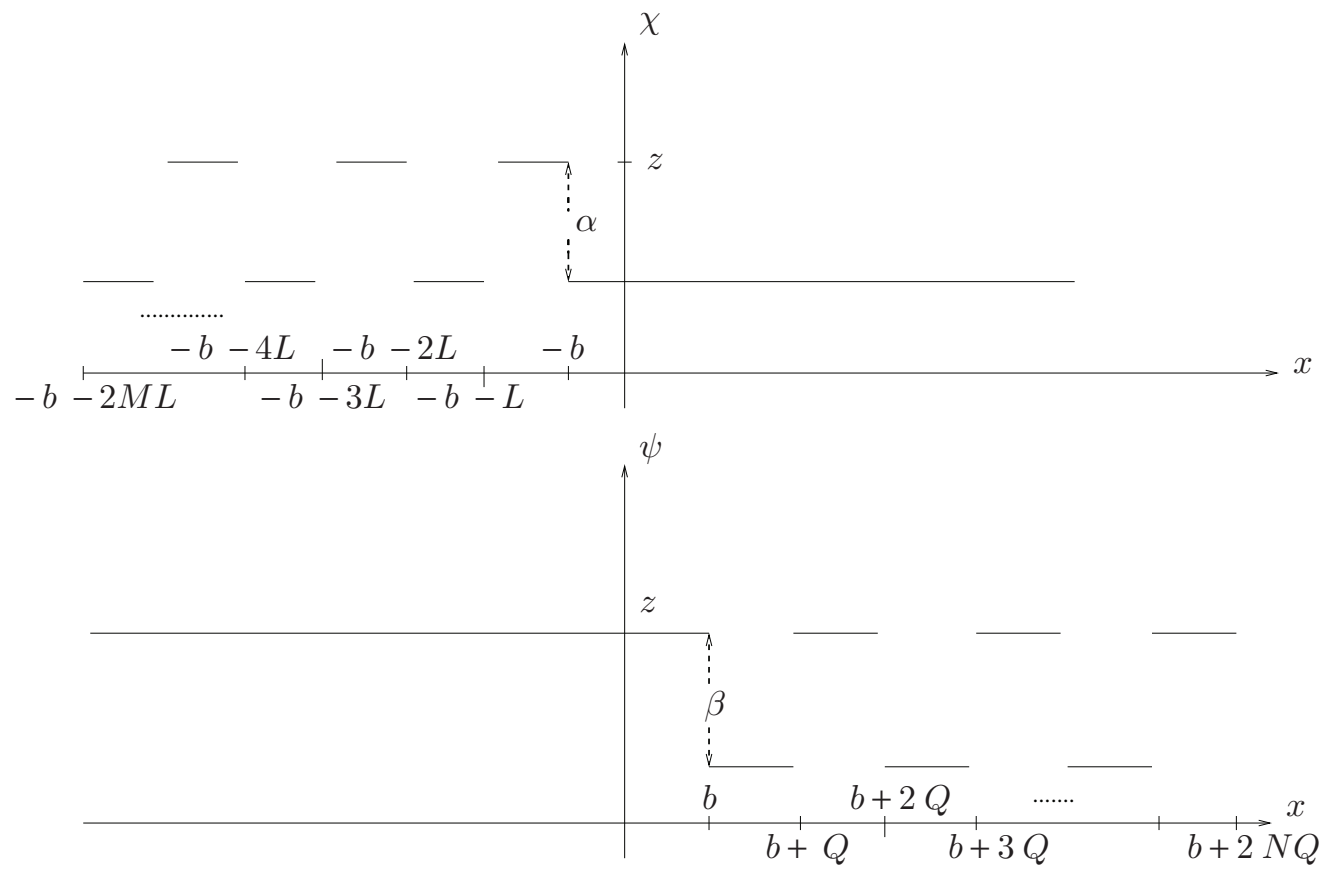

FIG. 3.1. Step functions $\chi$ and $\psi$.

For the sequence $\left\{r_{0 n}, s_{0 n}\right\}$ of initial data, we take

$$
\begin{gathered}
r_{0 n}=\chi\left(x ; \frac{\alpha}{(1+2 k)^{2 n N}}, b, \frac{L}{(1+2 k)^{2 n N}}, M(1+2 k)^{2 n N}\right), \\
s_{0 n}=\psi\left(x ; \frac{\beta}{n}, b, Q, n N\right),
\end{gathered}
$$

where $k$ is defined by (2.6), and the constants $\alpha, \beta, b, L, M, Q, N$ are such that

$$
\begin{aligned}
T V r_{0 n}+T V s_{0 n} & =2 M \alpha+2 N \beta=1, \\
2 L M+2 b & \leq \frac{\alpha_{2} Q}{\alpha_{1}-\alpha_{2}}, \\
\frac{2 k \alpha(\beta-\alpha)}{\beta+2 k \alpha}(1+2 k)^{N-1} & <\frac{2 k d \beta(1-d)}{1+2 k d}, \\
\alpha+\frac{2 k \alpha(\beta-\alpha)}{\beta+2 k \alpha}(2 k)^{-1}\left[(1+2 k)^{N-1}-1\right] & <\beta \frac{\sqrt{2 k^{2}+3 k+1}-k-1}{2 k^{2}+2 k},
\end{aligned}
$$

where

$$
d=\frac{\sqrt{2 k^{2}+3 k+1}-k-1}{2 k^{2}+2 k} .
$$

We have scaled the initial data so that their total variation remains fixed. Since $\sigma$ in (1.7) is invariant under rescaling of $u$, one may treat large data as small. It will suffice to describe $\left(r_{1}, s_{1}\right)$, as exhibited below, because $\left(r_{n}, s_{n}\right)$ may then be obtained by rescaling. 
In the solution we encounter interactions of 1-shocks with 2-shocks, of 1-shocks with 2rarefactions, and of 1-rarefactions with 2-shocks, as depicted by Figures 3.2, 3.3, and 3.6. respectively. We also have interactions of 1-rarefactions with 2-rarefactions, as depicted in Figures 3.4 and 3.5 .
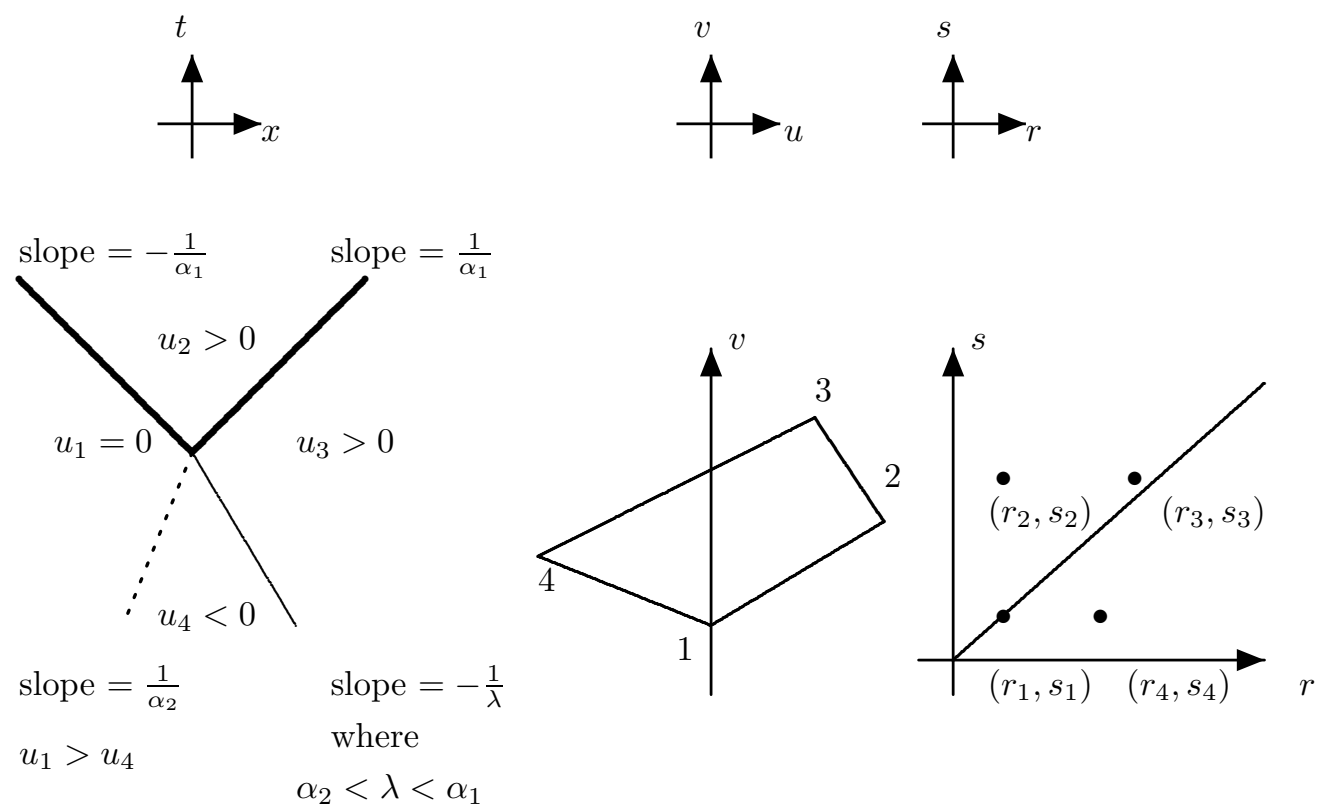

FIG. 3.2. Interaction of a 1-shock with a 2-shock.
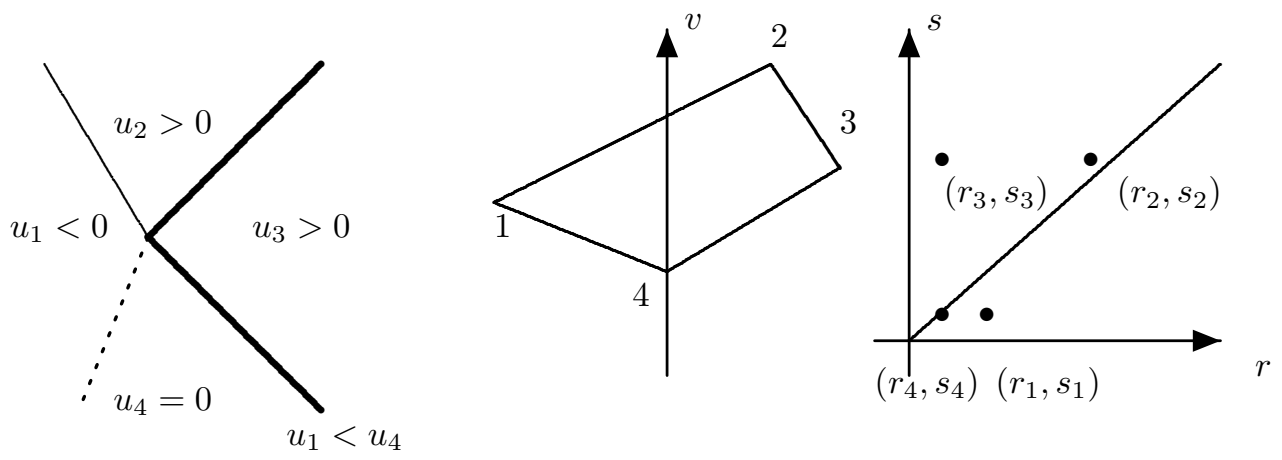

FIG. 3.3. Interaction of a 1-shock with a 2-rarefaction.

In the interaction depicted in Figure 3.2 the incoming wave strength is $\left|r_{1}-r_{3}\right|+\mid s_{3}-$ $s_{4} \mid$, the outgoing wave strength is $\left|r_{1}-r_{3}\right|+\left|s_{1}-s_{2}\right|$, and therefore there is no change in the wave strength. Similarly for the interactions depicted in Figures 3.4 3.5, and 3.6, the total wave strength of incoming and outgoing shocks remains the same. By contrast, in the interaction depicted in Figure 3.3. the incoming wave strength is $\left|r_{4}-r_{1}\right|+\left|s_{4}-s_{3}\right|$, 

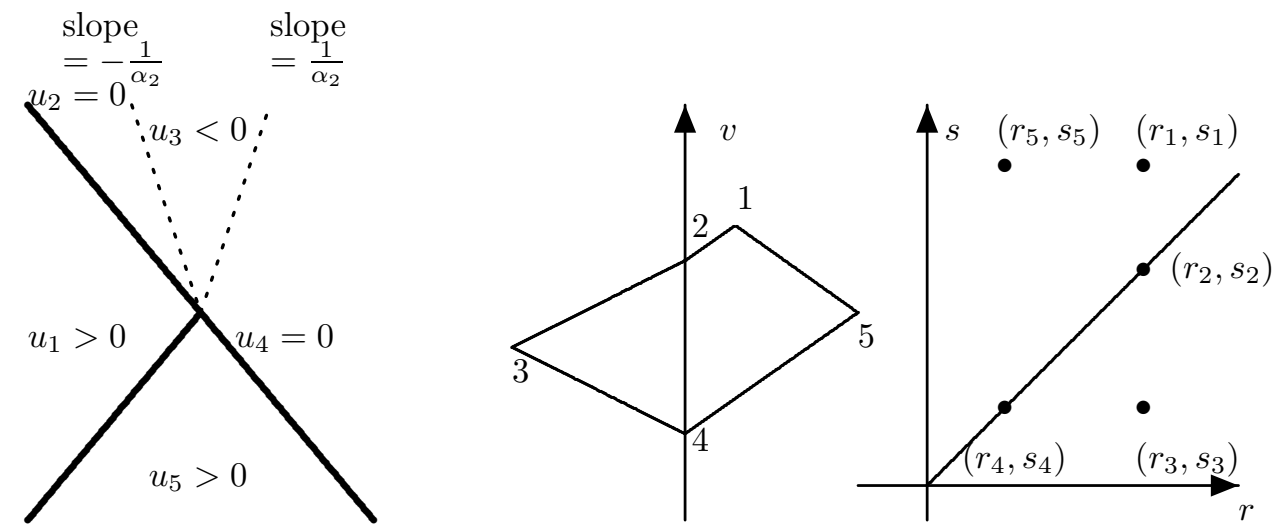

FIG. 3.4. Interaction of a 1-rarefaction with a 2-rarefaction.
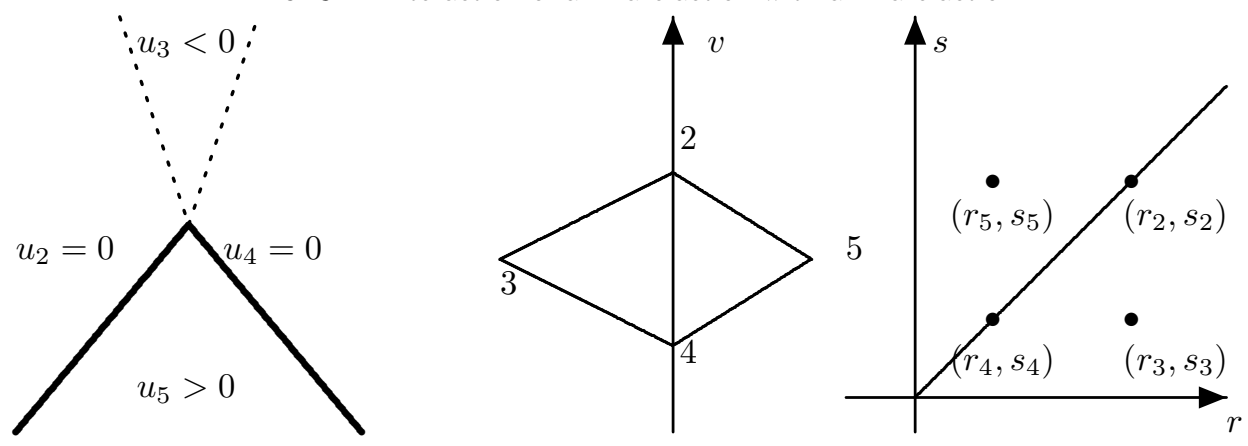

FIG. 3.5. Interaction of a 1-rarefaction with a 2-rarefaction.
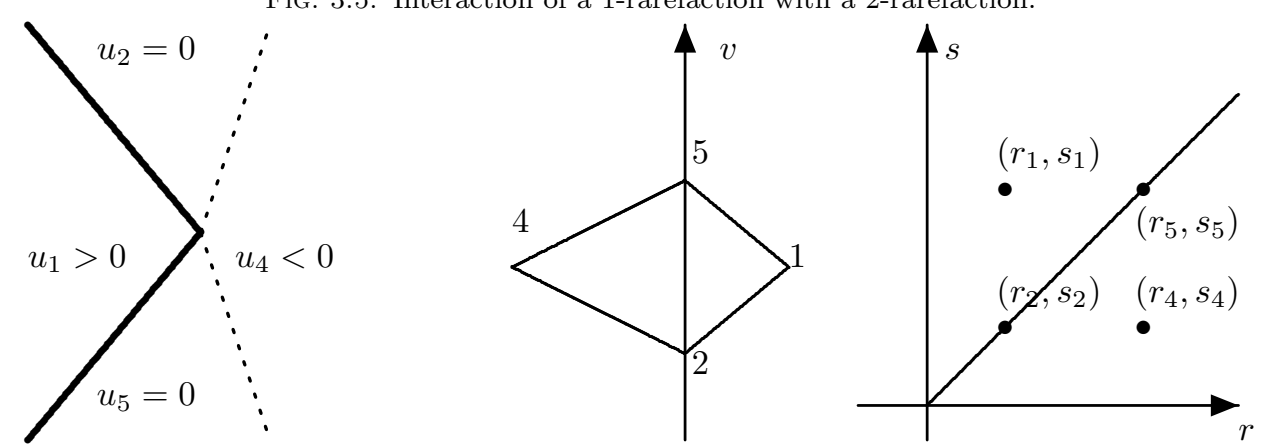

FIG. 3.6. Interaction of a 1-rarefaction with a 2-shock.

the outgoing wave strength is $\left|r_{1}-r_{4}\right|+2\left|r_{1}-r_{2}\right|+\left|s_{1}-s_{2}\right|$, and thus the wave strength increases by $2\left|r_{1}-r_{2}\right|$.

The resulting solution $\left(r_{1}, s_{1}\right)$ is depicted in Figure 3.7. Starting from the origin and moving to the left along the $x$-axis, we first encounter a 2-rarefaction generation point, then a 2-shock generation point, then a 2-rarefaction generation point and so on, until we end up with the $M$ th 2 -shock generation point. These waves issue from the $x$-axis, with speed $\alpha_{1}$ and at a distance $L$ from each other. Starting again from the origin but now 


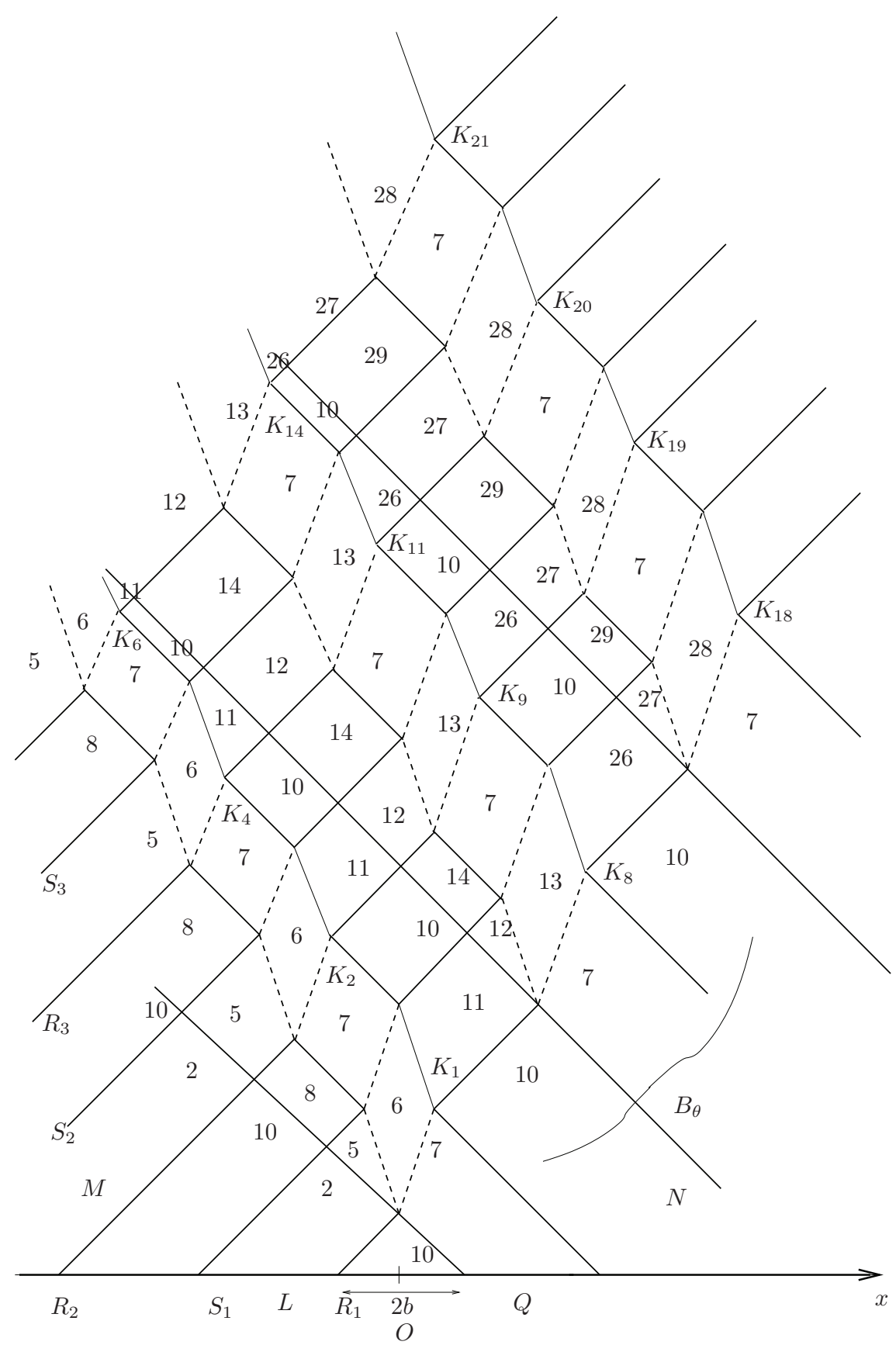

FIG. 3.7. Interaction pattern. The resulting solution $\left(r_{1}, s_{1}\right)$. 


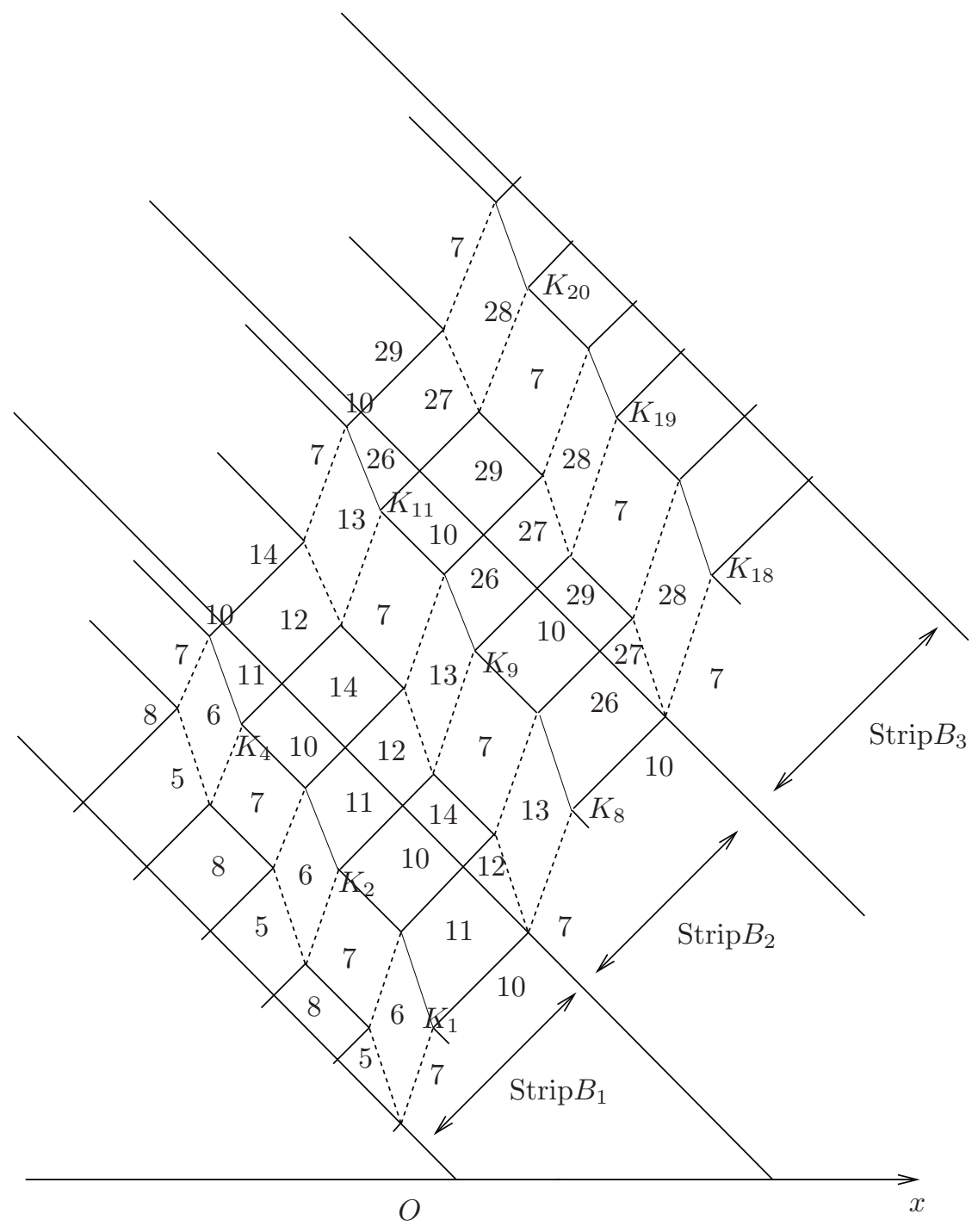

FIG. 3.8. Strips $B_{\theta}, \theta=1, \ldots, N$, in the upper half plane.

moving to the right along the $x$-axis, we first encounter a 1-rarefaction generation point, then a 1-shock generation point, then a 1-rarefaction generation point and so on, until we end up with the $N$ th 1 -shock generation point. These waves issue from the $x$-axis with speed $-\alpha_{1}$ and at a distance $Q$ from each other. Every 2 -wave, shock or rarefaction, originating from a point on the negative semi-axis propagates to the right and collides with each and every one of the $N$ 1-waves, but never collides with any one of the other 2 -waves. After the $N$ interactions are completed, the wave propagates undisturbed with constant speed $\alpha_{1}$. Similarly every 1-wave, shock or rarefaction, originating from a point on the positive semi-axis, propagates to the left and collides with each and every one of 


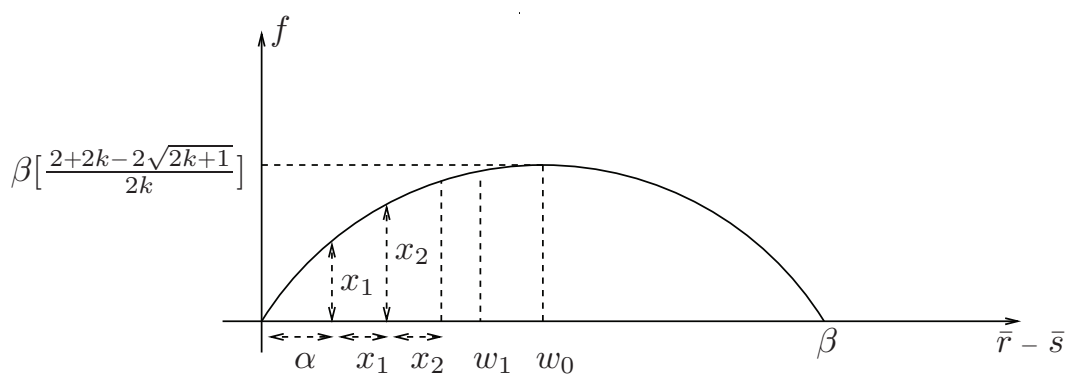

Fig. 3.9. Jump $f=\Delta r(\cdot, \bar{r}-\bar{s})$.

the $M$ 2-waves, but never collides with any of the other 1-waves (as we shall see, this holds under the assumption (3.2)). After the $M$ interactions with the 2 -waves are completed, the wave propagates undisturbed with constant speed $-\alpha_{1}$. All the interactions, $4 M \cdot N$ in number, occur before time $t_{1}$, where

$$
t_{1} \leq \frac{2 N Q+2 b}{\alpha_{2}}+\frac{2 M L+2 b}{\alpha_{2}} .
$$

Beyond time $t_{1}$, the total variation remains constant. To estimate the total variation of the solution $T V r_{1}\left(\cdot, t_{1}\right)+T V s_{1}\left(\cdot, t_{1}\right)$ at time $t_{1}$, we calculate the change in the wave strength at each interaction point. As noted above, we have change in the wave strength only at interactions of 1-shocks with 2-rarefactions; see Figure 3.3. The sum of all these changes is denoted by $V$. Therefore the total variation is greater than the sum of the total variation of the solution at $t=0, T V r_{1}(\cdot, 0)+T V s_{1}(\cdot, 0)$, plus $V$.

The next step is to estimate $V$. We divide the upper half-plane into strips $B_{\theta}, \theta=$ $1, \ldots, N$, as shown in Figure 3.8, constructed by the following procedure. The boundaries of the strips are generated by the 1-rarefaction shocks which emanate from the positive semi-axis and have slope $-\left(\alpha_{1}\right)^{-1}$. We let $V_{\theta}$ denote the change in the total variation in such a strip. Since $V=\sum_{\theta=1}^{N} V_{\theta}$, we need to estimate each $V_{\theta}$. From Figures 3.7 and 3.8, we see that $M$ interactions take place in each strip and each one yields the same amount of increase in the wave strength. Hence $V_{\theta}$ is $M$ times the amount of wave strength increase at the first interaction point at the right end of the strip $B_{\theta}$. We need the following preparation. Consider a 1-shock connecting the left state $\bar{u}<0$ and the right state $\underline{u}>0$, the corresponding Riemann invariants being $(\bar{r}, \bar{s})$ and $(\underline{r}, \underline{s})$. The jumps $\Delta r=\underline{r}-\bar{r}$ and $\Delta s=\underline{s}-\bar{s}$ are related by

$$
\Delta r=f(\Delta s, \bar{r}-\bar{s})=\frac{2 k(\bar{r}-\bar{s})[\Delta s-(\bar{r}-\bar{s})]}{\Delta s+2 k(\bar{r}-\bar{s})} .
$$

We keep $\Delta s$ fixed and equal to $\beta$, and then draw $f$ as a function of $\bar{r}-\bar{s}$; see Figure 3.9 , The slope of the tangent at $(0, f(\beta, 0)),\left(w_{1}, f\left(\beta, w_{1}\right)\right),\left(w_{0}, f\left(\beta, w_{0}\right)\right)$ and $(\beta, f(\beta, \beta))$, where

$$
w_{0}=\beta \frac{(\sqrt{2 k+1}-1)}{2 k}, \quad w_{1}=\beta\left[\frac{\sqrt{2 k^{2}+3 k+1}-k-1}{2 k^{2}+2 k}\right]=d \beta,
$$

is $2 k, k, 0$, and $-\frac{2 k}{2 k+1}$, respectively. In the solution, we select the initial data in such a way that $\Delta s=\beta$ and $\bar{r}-\bar{s} \leq w_{1}$ for each 1-shock that participates in the interactions 


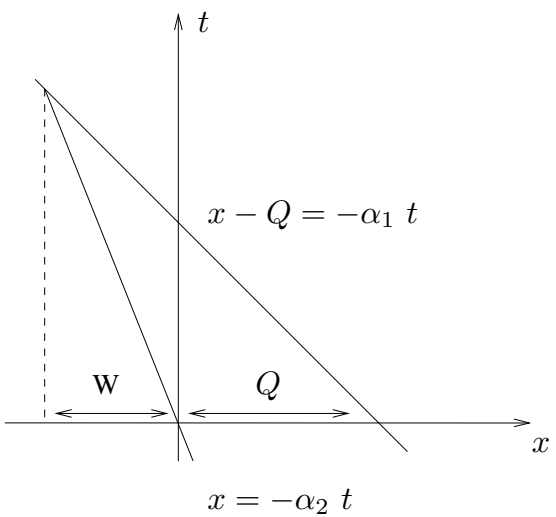

FIG. 3.10. Left-propagating waves.

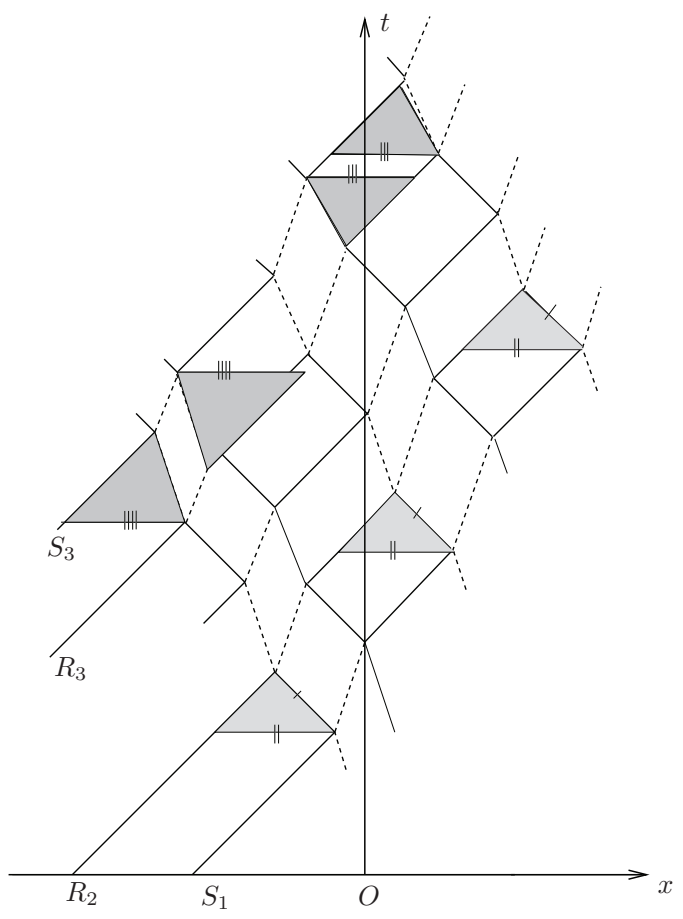

FIG. 3.11. Right-propagating waves.

with a 2-rarefaction wave, Figure 3.3. and is the first one we encounter at the right end of each strip $B_{\theta}$. This guarantees that the slope of the tangent at $(\bar{r}-\bar{s}, f(\beta, \bar{r}-\bar{s}))$ will lie between $k$ and $2 k$.

Figure 3.10 depicts the two straight lines $x-Q=-\alpha_{1} t$ and $x=-\alpha_{2} t$ which intersect at $x=-w$, where

$$
w=\frac{\alpha_{2} Q}{\alpha_{1}-\alpha_{2}} .
$$

To guarantee that the solution $\left(r_{1}, s_{1}\right)$ is as depicted in Figure 3.7 and the 1-waves never intersect with one another, we use Figures 3.10 and 3.11, and pick the constants $\alpha, \beta, b, L, M, Q$, and $N$ so that

$$
2 L M+2 b \leq \frac{\alpha_{2} Q}{\alpha_{1}-\alpha_{2}}
$$

and

$$
2 M \alpha+2 N \beta=1
$$

In what follows, we will use the function $f$ defined by (2.5). The 1-shock that participates in the interaction at the point $K_{1}$ (Figure 3.7), namely the first point at the right end of the strip $B_{1}$, has $\bar{r}-\bar{s}=\alpha, \Delta s=\beta$, and $\Delta r=x_{1}$, where $x_{1}=\frac{2 k \alpha(\beta-\alpha)}{\beta+2 k \alpha}$. The 1 -shock that participates in the interaction at the point $K_{8}$, namely the first point at the 

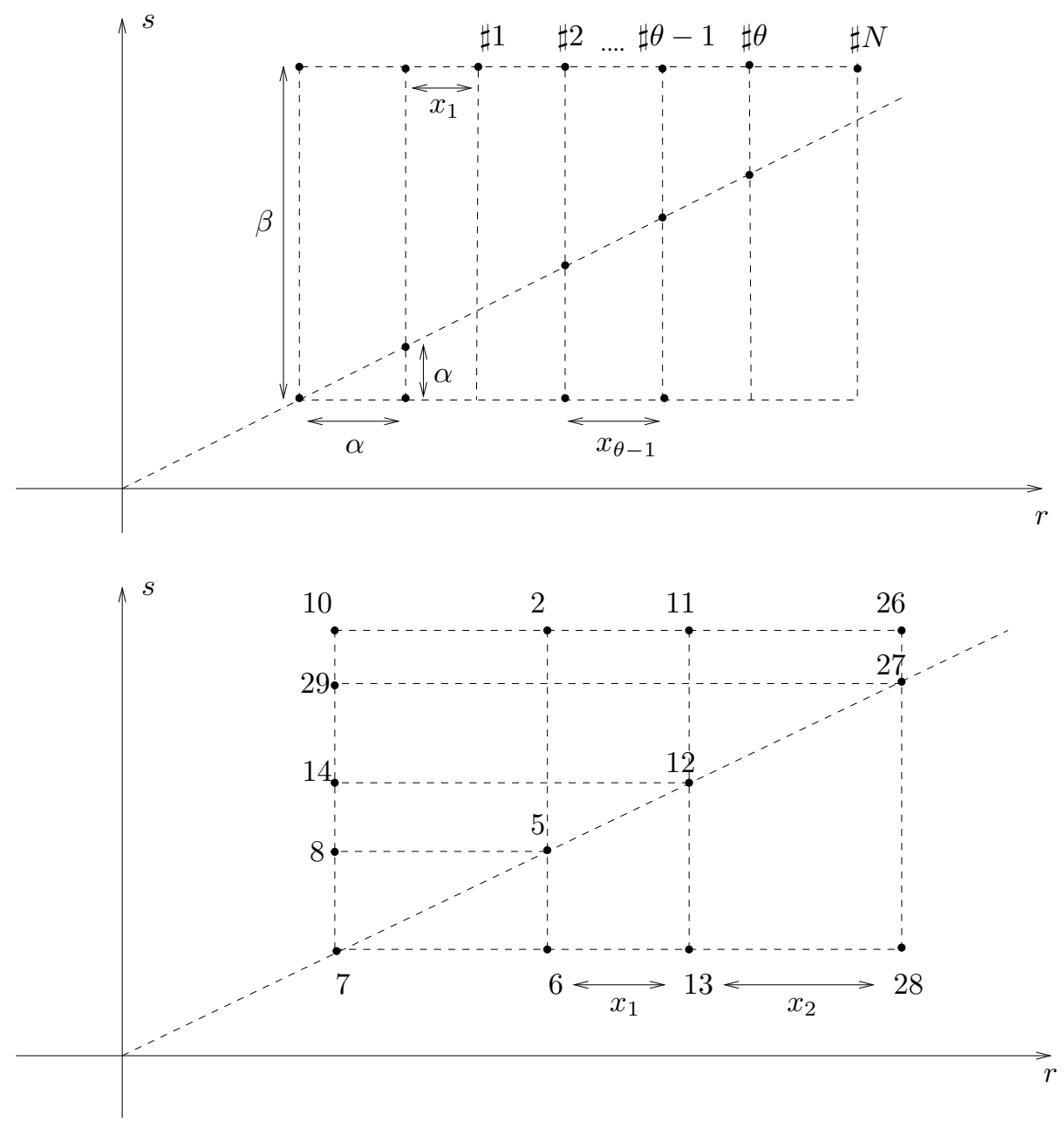

FIG. 3.12. Observations concerning right-propagating waves.

right end of the strip $B_{2}$, has $\bar{r}-\bar{s}=\alpha+x_{1}, \Delta s=\beta$, and $\Delta r=x_{2}$. From Figure 3.9 we can see that $x_{2}=f\left(\beta, \alpha+x_{1}\right)$. From the slopes we also obtain that $\frac{x_{2}-x_{1}}{x_{1}}=k_{1} \geq k$, so $x_{2}=x_{1}\left(1+k_{1}\right) \geq x_{1}(1+k)$. The 1 -shock that participates in the interaction at $K_{18}$, namely the first point at the right end of the strip $B_{3}$, has $\bar{r}-\bar{s}=\alpha+x_{1}+x_{2}, \Delta s=\beta$, and $\Delta r=x_{3}$, where $x_{3}=f\left(\beta, \alpha+x_{1}+x_{2}\right)$. From the slopes we see that $\frac{x_{3}-x_{2}}{x_{2}}=k_{2} \geq k$, and thus

$$
x_{3}=x_{2}\left(1+k_{2}\right)=x_{1}\left(1+k_{1}\right)\left(1+k_{2}\right) \geq x_{1}(1+k)^{2} .
$$

Since $f\left(w_{1}\right)=\frac{2 k d \beta(1-d)}{1+2 k d}$, and under the assumptions (3.3), (3.4), we get

$$
\begin{gathered}
x_{1}\left(1+k_{1}\right) \cdots\left(1+k_{N-1}\right) \leq x_{1}(1+2 k)^{N-1}<\frac{2 k d \beta(1-d)}{1+2 k d}, \\
\alpha+x_{1}+\cdots+x_{N-1} \leq \alpha+(2 k)^{-1} x_{1}\left[(1+2 k)^{N-1}-1\right]<w_{1} .
\end{gathered}
$$


We follow the same idea for the 1-shock that participates in the first interaction at the right end of the strips $B_{4}, \ldots, B_{\theta}$. Therefore, the 1-shock that participates in the interaction at the first point at the right end of the strip $B_{\theta}$ has $\bar{r}-\bar{s}=\alpha+x_{1}+x_{2}+$ $\cdots+x_{\theta-1}, \Delta s=\beta$, and $\Delta r=x_{\theta}$, where $x_{\theta}=f\left(\beta, \alpha+x_{1}+x_{2}+\cdots+x_{\theta-1}\right)$. From the slopes we will have

$$
x_{\theta}=x_{1}\left(1+k_{1}\right) \cdots\left(1+k_{\theta-1}\right) \geq x_{1}(1+k)^{\theta-1} .
$$

Thus, starting from a 1-shock where $\bar{r}-\bar{s}=\alpha$ and $\Delta r=x_{1}$, and moving in the "northeasterly" direction, for $\theta=2, \ldots, N$, we arrive at a 1-shock with $\bar{r}-\bar{s}=\alpha+x_{1}$ and $\Delta r=x_{2}$ and eventually to a 1 -shock with $\bar{r}-\bar{s}=\alpha+x_{1}+x_{2}+\cdots+x_{N-1}$ and $\Delta r=x_{N}$.

Since our goal is to estimate the change in the total variation for each strip $B_{\theta}$, we focus our attention to this region and after some calculations we obtain, from Figure 3.12 ,

$$
\left|r_{6}-r_{11}\right|=x_{1}, \quad\left|r_{13}-r_{26}\right|=x_{2} .
$$

Using Figure 3.7, we estimate the change in strength for each interaction in the strip $B_{\theta}$ and obtain the following inequality:

$$
V_{\theta} \geq 2 M x_{1}(1+k)^{\theta-1} .
$$

We denote by $I_{K}$ the increase in the wave strength at the point $K$. When we check all interaction points we conclude that for the first term of the sequence of solutions $\left(r_{1}, s_{1}\right)$,

$$
\begin{aligned}
t_{1} \leq & \frac{2 N Q+2 b}{\alpha_{2}}+\frac{2 M L+2 b}{\alpha_{2}} \\
T V_{t_{1}} r_{1}+T V_{t_{1}} s_{1}= & T V r_{01}+T V s_{01}+I_{K_{1}}+I_{K_{2}} \\
& +I_{K_{4}}+I_{K_{6}}+I_{K_{8}}+I_{K_{9}}+I_{K_{11}} \\
& +I_{K_{14}}+I_{K_{18}}+I_{K_{19}}+I_{K_{20}}+I_{K_{21}}+\cdots
\end{aligned}
$$

Therefore we have

$$
\begin{aligned}
T V_{t_{1}} r_{1}+T V_{t_{1}} \quad s_{1} & \geq T V r_{01}+T V s_{01}+\sum_{\theta=1}^{N} V_{\theta} \\
& \geq T V r_{01}+T V s_{01}+2 M x_{1} \sum_{\theta=1}^{N}(1+k)^{\theta-1}
\end{aligned}
$$

To define $\left\{\left(r_{n}, s_{n}\right)\right\}$ for $n=2,3, \ldots$, let $\mu_{n}$ be the integer part of $(1+2 k)^{2 n N}$; we then keep $Q, b$ fixed, we change $N$ to $n N, \beta$ to $\frac{\beta}{n}, \alpha$ to $\frac{\alpha}{\mu_{n}}, L$ to $\frac{L}{\mu_{n}}, M$ to $\mu_{n} \cdot M$, and $x_{1}$ to $\frac{x_{1}}{\mu_{n}}$. Thus we get

$$
T V r_{0 n}+T V s_{0 n}=2 M \alpha+2 N \beta
$$

and for

$$
t_{n} \leq \frac{2 N n Q+2 b}{\alpha_{2}}+\frac{2 M L+2 b}{\alpha_{2}}
$$




$$
\begin{aligned}
T V_{t_{n}} \quad r_{n}+T V_{t_{n}} \quad s_{n} & \geq T V r_{0 n}+T V s_{0 n}+2 M x_{1} \sum_{\theta=1}^{N n}(1+k)^{\theta-1} \\
& \geq\left(1+\frac{1}{2 M \alpha+2 N \beta} \tilde{c_{n}}\right) \cdot\left\{T V r_{0 n}+T V s_{0 n}\right\} \\
& \geq c_{n} \cdot\left\{T V r_{0 n}+T V s_{0 n}\right\}=c_{n},
\end{aligned}
$$

where

$$
\tilde{c_{n}}=2 M x_{1} \sum_{\theta=1}^{N n}(1+k)^{\theta-1}, \quad c_{n}=1+\frac{1}{2 M \alpha+2 N \beta} \tilde{c_{n}} .
$$

The series $\lim _{n \rightarrow \infty} \tilde{c_{n}}$ is divergent. Thus, $\operatorname{TV} r_{n}\left(\cdot, t_{n}\right)+T V s_{n}\left(\cdot, t_{n}\right)>c_{n}$, where $c_{n} \rightarrow \infty$.

Therefore we notice that we have a violation of the Glimm estimate (1.3).

\section{REFERENCES}

[1] P. Baiti and H.K. Jenssen, Blowup in $L^{\infty}$ for a class of genuinely nonlinear hyperbolic systems of conservation laws. Discrete Contin. Dynam. Systems 7 (2001), 837-853. MR.1849664|(2003m:35155)

[2] S. Bianchini and A. Bressan, Vanishing viscosity solutions of nonlinear hyperbolic systems. Ann. of Math. 161 (2005), 1-120. MR2150387 (2007i:35160)

[3] A. Bressan, Hyperbolic System of Conservation Laws. The One Dimensional Cauchy Problem. Oxford University Press, 2000. MR.1816648 (2002d:35002)

[4] C.M. Dafermos, Hyperbolic Conservation Laws in Continuum Physics, Grundlehren Math. Wissenschaften Series, Second Edition, Springer Verlag, 325, 2005. MR2169977 (2006d:35159)

[5] C.M. Dafermos, Polygonal approximations of solutions of the initial value problem for a conservation law. J. Math. Anal. Appl. 38 (1972), 33-41. MR0303068 (46:2210)

[6] J. Glimm, Solutions in the large for nonlinear hyperbolic systems of equations. Comm. Pure Appl. Math. 18 (1965), 697-715. MR0194770 (33:2976)

[7] J. Glimm and P.D. Lax, Decay of solutions of systems of nonlinear hyperbolic conservation laws. Memoirs AMS, No. 101 (1970). MR0265767 (42:676)

[8] H. Holden and N.H. Risebro, Front Tracking for Hyperbolic Conservation Laws. New York: Springer, 2002. MR 1912206 (2003e:35001)

[9] A. Jeffrey, Breakdown of the solution to a completely exceptional system of hyperbolic equations. J. Math. Anal. Appl. 45 (1974), 375-381. MR0333472 (48:11797)

[10] H.K. Jenssen, Blowup for systems of conservation laws. SIAM J. Math. Anal. 31 (2000), 894-908. MR 1752421 (2001a:35114)

[11] J.-L. Joly, G. Metivier, and J. Rauch, A nonlinear instability for $3 \times 3$ systems of conservation laws. Comm. Math. Phys. 162 (1994), 47-59. MR1272766 (95f:35145)

[12] S. Kruzkov, First-order quasilinear equations with several space variables. Mat. Sbornik 123 (1970), 228-255. English translation: Math. USSR Sbornik 10 (1970), 217-273. MR0267257 (42:2159)

[13] T.-P. Liu, Admissible solutions of hyperbolic conservation laws. Memoirs AMS 30 (1981), No. 240. MR603391 (82i:35116)

[14] T.-P. Liu, Z. Xin and T. Yang, Vacuum states for compressible flow. Discrete Contin. Dynam. Systems 4 (1998), no. 1, 1-32. MR1485360 (98k:76116)

[15] J. Smoller, Shock Waves and Reaction-diffusion Equations, Springer, New York, 1994. MR1301779 (95g:35002)

[16] K. Trivisa, A priori estimates in hyperbolic systems of conservation laws via generalized characteristics. Comm. PDE 22 (1997), 235-267. MR.1434145 (98a:35086)

[17] K. Trivisa, BV estimates for $n \times n$ systems of conservation laws. Contemp. Math. 327 (2003), 341358. MR1991553(2004i:35222)

[18] C. Tsikkou, BV Estimates for the P-System, preprint.

[19] R. Young, On elementary interactions for hyperbolic conservation laws, preprint.

[20] R. Young, Isentropic gas dynamics with large data. In Hyperbolic Problems: Theory, Numerics, Applications, T. Hou and E. Tadmor, eds., pp. 929-939. Springer, 2003. MR2053240

[21] R. Young, Blowup of solutions and boundary instabilities in nonlinear hyperbolic equations. Comm. Math. Sci. 1 (2003), 269-292. MR.1980476 (2004j:35190) 
[22] R. Young and W. Szeliga, Blowup with small BV data in hyperbolic conservation laws. Arch. Ration. Mech. Anal. 179, No. 1, 31-54 (2006). MR2208288 (2007h:35228) 